\title{
Final Results from HERMES on Hadronization in Nuclear Environment
}

\author{
Z. Akopov ${ }^{1} *$ \\ Yerevan Physics Institute, 375036 Yerevan, Armenia
}

\begin{abstract}
A series of semi-inclusive deep-inelastic scattering measurements on deuterium, helium, neon, krypton, and xenon targets has been performed in order to study hadronization. The data were collected with the HERMES detector at the DESY laboratory using a $27.6 \mathrm{GeV}$ positron or electron beam. Hadron multiplicities on nucleus $A$ relative to those on the deuteron, $R_{A}^{h}$, are presented for various hadrons $\left(\pi^{+}, \pi^{-}, \pi^{0}, K^{+}\right.$, $K^{-}, p$, and $\bar{p}$ ) as a function of the virtual-photon energy $\nu$, the fraction $z$ of this energy transferred to the hadron, the photon virtuality $Q^{2}$, and the hadron transverse momentum squared $p_{t}^{2}$. The data reveal a systematic decrease of $R_{A}^{h}$ with the mass number $A$ for each hadron type $h$. For pions two-dimensional distributions also are presented. These indicate that the dependences of $R_{A}^{\pi}$ on $\nu$ and $z$ can largely be described as a dependence on a single variable $L_{c}$, which is a combination of $\nu$ and $z$. The dependence on $L_{c}$ suggests in which kinematic conditions partonic and hadronic mechanisms may be dominant.
\end{abstract}

\section{Introduction}

Understanding the confinement of quarks and gluons in hadrons still is one of the great challenges in hadronic physics. To uncover its nature, hadronic reactions in a nuclear medium, either cold or hot, are studied. Typical examples are the measurements of hadron production on nuclear targets in semi-inclusive deep-inelastic scattering of leptons $[2,3,4]$ and the jet-quenching and parton energy-loss phenomena observed in ultra-relativistic heavyion collisions [5]. In each case hadron yields are observed that are different from those observed in the corresponding reactions on free nucleons. The process that leads from the partons produced in the elementary interaction to the hadrons observed experimentally is commonly referred to as hadronization or fragmentation. According to theoretical estimates the hadronization process occurs over length scales varying from less than a femtometer to several tens of femtometers. At these length scales the magnitude of the strong coupling constant is such that perturbative techniques cannot be applied. Hence, hadronization is an intrinsically non-perturbative QCD process, for which only approximate theoretical approaches are presently available. Experimental data are vital for supporting these theoretical developments, since they can be used to gauge or guide the calculations.

The hadronization process in a nuclear medium can be studied by means of semi-inclusive deep-inelastic scattering (SIDIS) of electrons or positrons from nuclei. For that purpose the multiplicity ratio $R_{A}^{h}$ is introduced, which is defined as the ratio of the number of hadrons $h$ produced per deep-inelastic scattering (DIS) event on a nuclear target with mass number $A$ to that for a deuterium (D) target:

$$
R_{A}^{h}\left(\nu, Q^{2}, z, p_{t}^{2}\right)=\frac{\left(\frac{N^{h}\left(\nu, Q^{2}, z, p_{t}^{2}\right)}{N^{e}\left(\nu, Q^{2}\right)}\right)_{A}}{\left(\frac{N^{h}\left(\nu, Q^{2}, z, p_{t}^{2}\right)}{N^{e}\left(\nu, Q^{2}\right)}\right)_{D}},
$$

* On behalf of the HERMES Collaboration 


\section{Experiment and Data Analysis}

The measurements were performed with the HERMES spectrometer [6] using a $27.6 \mathrm{GeV}$ positron or electron beam stored in the HERA ring at DESY. Typical beam currents were $40 \mathrm{~mA}$ down to $5 \mathrm{~mA}$. The scattered leptons and the produced hadrons were detected within
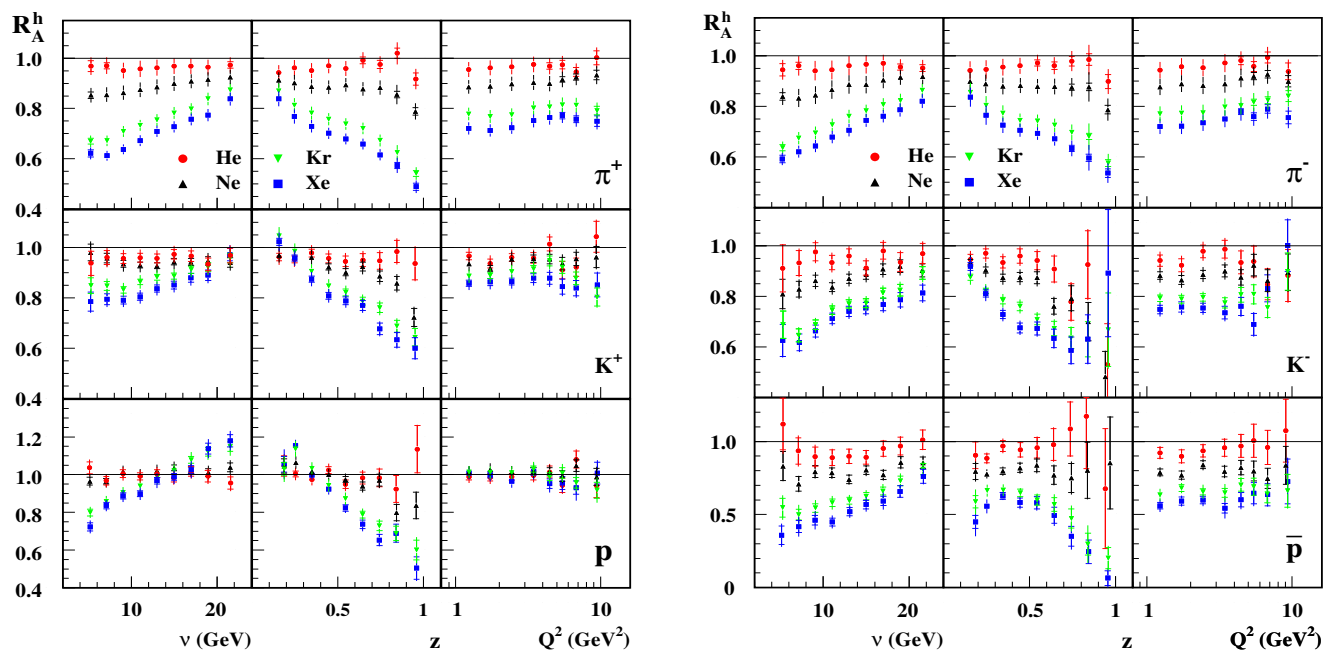

Figure 1: Values of $R_{A}^{h}$ for positively and negatively charged hadrons as a function of $\nu, z$, and $Q^{2}$.

an angular acceptance of $\pm 170 \mathrm{mrad}$ horizontally and $\pm(40-140) \mathrm{mrad}$ vertically. The lepton trigger was formed by a coincidence between signals from three scintillator hodoscope planes and a lead-glass calorimeter. A minimum energy deposit in the latter of $3.5 \mathrm{GeV}(1.4 \mathrm{GeV})$ for unpolarized (polarized) target runs was required. The data were collected during the years 1999, 2000, 2004, and 2005, using unpolarized nuclear (He, Ne, Kr, Xe) and polarized or unpolarized deuterium (D) gaseous targets internal to the storage ring. The identification of charged pions, kaons, protons, and antiprotons is accomplished using the information from the Ring-Imaging Cerenkov detector (RICH) [7] The hadron multiplicity ratio $R_{A}^{h}$ as defined in Eq. 1 was determined as a function of the leptonic $\left(Q^{2}\right.$ and $\left.\nu\right)$ and hadronic $\left(z\right.$ and $\left.p_{t}^{2}\right)$ variables for all identified particles and all targets. The kinematic constraints imposed on the scattered leptons were identical for all analysed data:

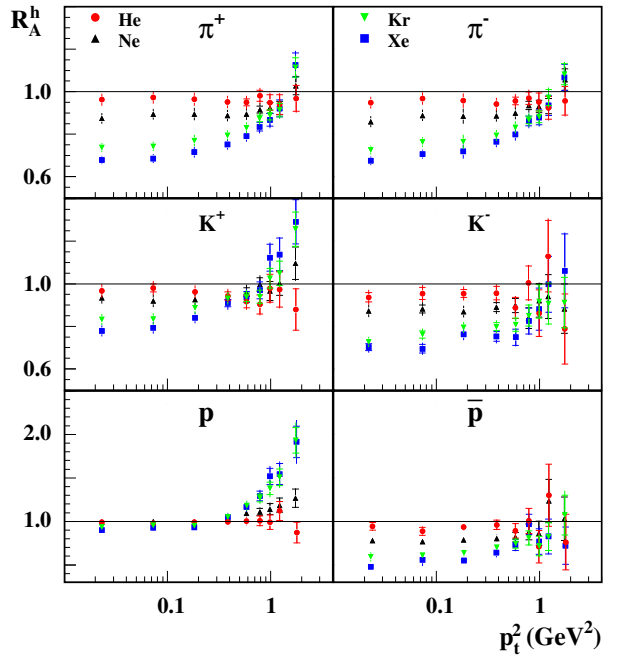

Figure 2: Values of $R_{A}^{h}$ for positively (left panel) and negatively (right panel) charged hadrons as a function of $p_{t}^{2}$. 
$Q^{2}>1 \mathrm{GeV}^{2}, W=\sqrt{2 M \nu+M^{2}-Q^{2}}>$

$2 \mathrm{GeV}$ (where $M$ is the nucleon mass) for the invariant mass of the photon-nucleon system, and $y=\nu / E<0.85$ for the energy fraction of the virtual photon. The constraints on $W$ and $y$ were applied in order to exclude nucleon resonances and to limit the magnitude of the radiative corrections to $R_{A}^{h}$, respectively. The resulting value of $x_{B j}=Q^{2} / 2 M \nu$ ranged from 0.023 to 0.8 .

\section{Results}

The experimental results are presented as dependences of the multiplicity ratios $R_{A}^{h}$ on the various kinematic variables and the mass number $A$ of the nucleus. The dependence of $R_{A}^{h}$ on $\nu, z, Q^{2}$, and $p_{t}^{2}$ for the various nuclei for all identified hadrons is shown in Figs. 12 The most prominent features of the data are an increased attenuation (decrease of $R_{A}^{h}$ below unity) with increasing value of the mass number $A$ of the nucleus and the attenuation becoming smaller (larger) with increasing values of $\nu(z), R_{A}^{h}$ dropping below 0.5 for xenon in some kinematic regions. At low values of $z$, especially for heavier targets and for protons and $K^{+}$particles, a strong rise of $R_{A}^{h}$, even to above unity, is observed. Presumably this is due to hadronic rescattering, where a higher energy particle through nuclear reactions produces one or more lower-energy particles. By combining the data for $\pi^{+}$and $\pi^{-}$, the dependence of $R_{A}^{\pi}$ on two of the variables $\nu, z, Q^{2}$, and $p_{t}^{2}$ together was investigated, and the results are shown in Fig. 3. The dependence on $Q^{2}$ depends weakly but noticeably
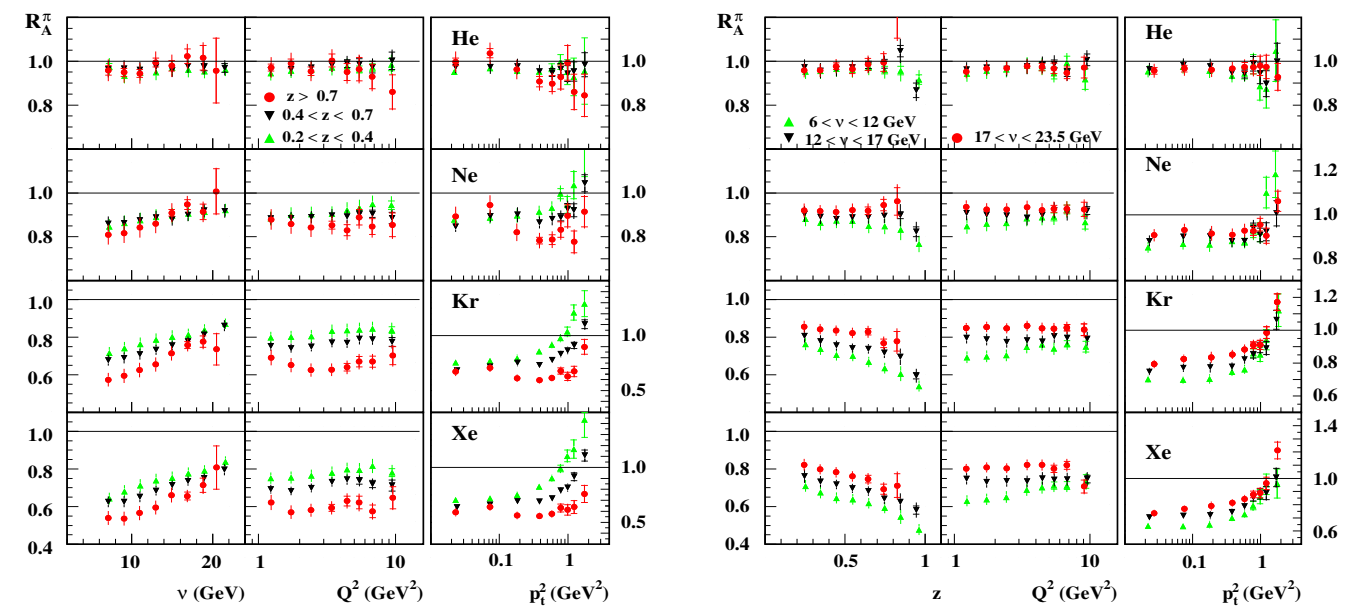

Figure 3: Values of $R_{A}^{h}$ for charged pions for three $z$ ranges and three $\nu$ ranges.

on the value of $\nu$, but practically not on that of $z$. The dependence on $p_{t}^{2}$ hardly depends on $\nu$ and $z$, except for the disapperance of the rise at large $p_{t}^{2}$ at $z \rightarrow 1$ mentioned above. However, the dependences on $\nu$ and $z$ are related. It was found that most of the dependence on $\nu$ and $z$ can be incorporated in a dependence on the combination $L_{c}=z^{0.35}(1-z) \nu / \kappa$, where $\kappa$ is the string tension in string models, which thus acts as a scaling variable. 
Since this function is close to the one given in the Lund model for the average formation length of a particle, by inspecting the value of $R_{A}^{\pi}$ vs. $L_{c}$ for the four nuclei, regions can tentatively be identified, where hadronic (absorption) plus partonic mechanisms are important, and a region at higher $L_{c}$ where only or mainly partonic mechanisms play a role.

In order to investigate this, values of $R_{A}^{\pi}$ versus $L_{c}$ for various values of $\nu$ and $z$ are shown in Fig. 4. Here $L_{c}$ is defined as:

$$
L_{c}=f(z) \frac{\nu}{\kappa}
$$

where $f(z)=z^{0.35}(1-z)$ and $\kappa=$ $1 \mathrm{GeV} / \mathrm{fm}$. This form for $f(z)$ is a convenient parametrization obeying the constraints at $z \rightarrow 0$ and $z \rightarrow 1$, and gives values for $L_{c}$ as a function of $z$ closely resembling the ones obtained with the Lund model.

A clear correlation can be observed between the values of $R_{A}^{h}$ and $L_{c}$, with only a relatively small residual spread at any fixed

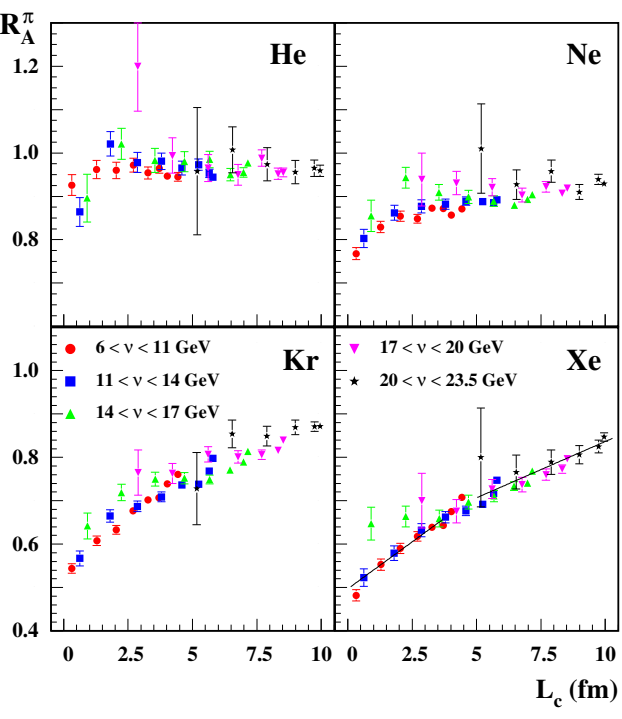

Figure 4: Values of $R_{A}^{\pi}(\nu, z)$ for $\mathrm{He}, \mathrm{Kr}, \mathrm{Ne}$, and $\mathrm{Xe}$ as a function of the variable $L_{c}$, see Eq. 2. The various $\nu$-bins are indicated by different symbols. Within the same $\nu$ bin the $z$ bins used are $0.2-0.3-0.4-0.5-0.6-$ $0.7-0.8-0.9-1.0$.

value of $L_{c}$. Evidently most of the dependence of $R_{A}^{h}$ on $\nu$ or $z$ in Fig. 3 can be described as a dependence on $L_{c}$, which thus acts as a scaling variable.

\section{Conclusions}

In total a very extensive data set to guide modeling hadronization in nuclear matter has been collected by the HERMES collaboration. A full theoretical description of hadronization in nuclei in one consistent framework, including partonic and hadronic (absorption plus rescattering) mechanisms is badly needed. Clearly it will be a challenge for any theoretical model that is developed to describe these data for the various hadrons and nuclei as a function of all kinematic variables, but if successful, this combination of data and theoretical interpretation will contribute essentially to the understanding of non-perturbative QCD at normal, and thence higher densities.

\section{References}

[1] Slides: http://indico. cern. ch/contributionDisplay . py? contribId=187\&sessionId=6\&conf Id=9499

[2] L. Osborne et al., Phys. Rev. Lett. 40 (1978) 1624.

[3] A. Airapetian et al. [HERMES Collaboration], Eur. Phys. J. C20 (2001) 479.

[4] A. Airapetian et al. [HERMES Collaboration], Phys. Lett. B577 (2003) 37.

[5] J. Adams et al. [STAR Collaboration], Phys. Rev. Lett. 92 (2004) 112301.

[6] K. Ackerstaff et al. [HERMES Collaboration], Nucl. Instr. and Meth. A417 (1998) 230.

[7] N. Akopov et al., Nucl. Instr. and Meth. A479 (2002) 511. 